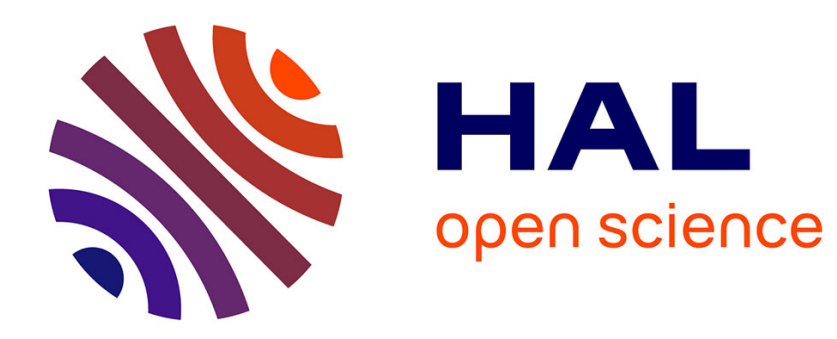

\title{
Numerical simulation of SiGe HBT's at cryogenic temperatures
}

\author{
D. Richey, J. Cressler, R. Jaeger
}

\section{To cite this version:}

D. Richey, J. Cressler, R. Jaeger. Numerical simulation of SiGe HBT's at cryogenic temperatures. Journal de Physique IV Proceedings, 1994, 04 (C6), pp.C6-127-C6-132. 10.1051/jp4:1994620 . jpa00253114

\section{HAL Id: jpa-00253114 https://hal.science/jpa-00253114}

Submitted on 1 Jan 1994

HAL is a multi-disciplinary open access archive for the deposit and dissemination of scientific research documents, whether they are published or not. The documents may come from teaching and research institutions in France or abroad, or from public or private research centers.
L'archive ouverte pluridisciplinaire HAL, est destinée au dépôt et à la diffusion de documents scientifiques de niveau recherche, publiés ou non, émanant des établissements d'enseignement et de recherche français ou étrangers, des laboratoires publics ou privés. 


\title{
Numerical simulation of SiGe HBT's at cryogenic temperatures
}

D.M. Richey, J.D. Cressler and R.C. Jaeger

Alabama Microelectronics Science and Technology Center, Electrical Engineering Department, 200 Broun Hall, Auburn University, AL 36849, U.S.A.

\begin{abstract}
This paper describes SCORPIO, a new one-dimensional, drift-diffusion simulator for modeling silicon-germanium heterojunction bipolar transistors (SiGe HB'T's) over a wide temperature range (77-400K). SCORPIO will be used to investigate fundamental low-temperature device physics problems and key device design issues. Comparisons of simulation results with experimental measurements are being used to ensure accurate model calibration.
\end{abstract}

\section{THE DEVICE SIMULATOR}

SCORPIO (Simulator for Cryogenic Research and SiGe Bipolar Device Optimization) is a newly developed device simulation program for investigating SiGe HBT behavior over a wide temperature range, including (but not limited to) liquid nitrogen temperature (LNT=77K). A simulation tool such as this not only allows for greater understanding of the underlying device physics, but also makes possible the optimization of devices for specific applications. SCORPIO uses a one-dimensional, finite difference, drift-diffusion approximation to model the bipolar device being simulated [1]. The one-dimensional structure used by SCORPIO is shown in Figure 1. Low temperature semiconductor device simulation is a difficult problem because parameters often assumed constant in room temperature ( $R T=300 \mathrm{~K}$ ) simulators may actually be complex functions of temperature (mobility, for example) [2]. Phenomena unique to low temperature operation, such as carrier freezeout, are typically not accounted for in simulators designed for room temperature use. In addition, the system of equations to be solved at liquid nitrogen temperature is much more ill-conditioned numerically than for room temperature, due to terms having exponential temperature dependencies. For these reasons, typical simulation programs have difficulty converging to a solution atLNT.

SCORPIO has been written in the $\mathrm{C}++$ programming language [3]. Object-oriented programming techniques have been used to simplify the internal representation of data structures such as vectors and matrices. The resulting source code is concise, readable, easy to maintain, and extremely modular. SCORPIO's modularity allows for "plug-in/plug-out" physics, in which modules for simulating various device phenomena may be turned on or off from one simulation to the next. In this way, one can see the differences in using Fermi-Dirac statistics instead of Boltzmann statistics, for example; or compare several different mobility models to see which one agrees most closely with experimental data. This flexibility has been extremely beneficial with respect to calibrating simulation results to measured data because it has allowed numerous models for each parameter to be easily tested in various combinations. 


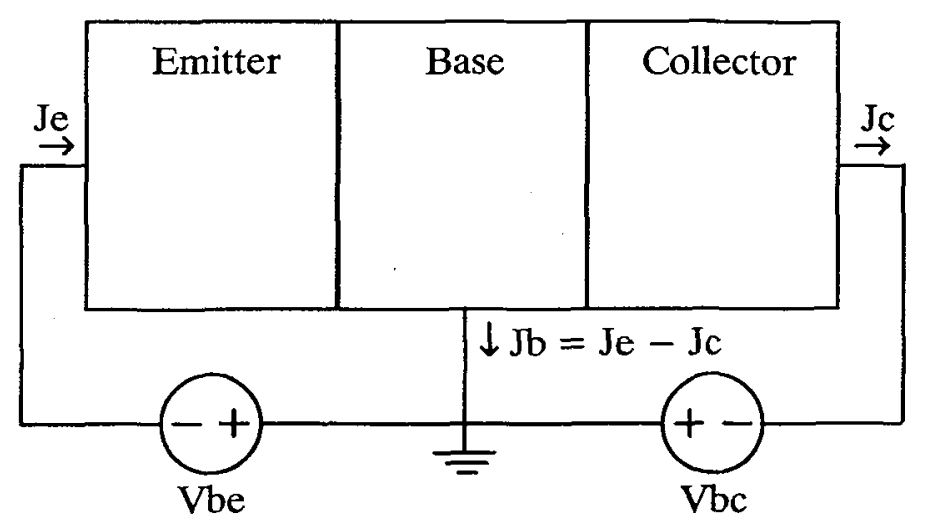

Figure 1. One-dimensional bipolar transistor device model.

\subsection{Semiconductor Device Equations}

To accurately analyze an arbitrary semiconductor device under various operating conditions, a mathematical model has to be given. The equations which form this model in SCORPIO are commonly called the drift-diffusion equations [4]. SCORPIO attempts to solve Poisson's equation and the electron and hole continuity equations simultaneously for the electrostatic potential $(\psi)$, the electron concentration $(n)$, and the hole concentration $(p)$ as functions of position at a specific temperature. Since these dependent variables $(\psi$, $n, p$ ) are of greatly differing orders of magnitude, the first step towards numerical analysis has to be an appropriate parameter scaling [5], [6]. For the steady-state condition, the scaled (unitless) equations may be written as:

$$
\begin{aligned}
& \nabla^{2} \psi=n-p-N_{D}^{+}+N_{A}^{-} \\
& \nabla \cdot J_{n}=R \\
& \nabla \cdot J_{p}=-R \\
& J_{n}=-\mu_{n}\left(n \nabla \psi_{n}-\nabla n\right) \\
& J_{p}=-\mu_{p}\left(p \nabla \psi_{p}+\nabla p\right) \\
& \psi_{n}=\psi+\chi+\ln \left(\gamma_{n} N_{c}\right) \\
& \psi_{p}=\psi+\chi+E_{g}-\ln \left(\gamma_{p} N_{y}\right)
\end{aligned}
$$

The scaled equations are attractive from a computational point of view because they are simpler, and no operations with constants are involved in their evaluation. Note that while (4) and (5) closely resemble the current relations usually used to describe homojunction devices, this particular formulation allows for the bandgap energy $\left(E_{g}\right)$, electron affinity $(\chi)$, and densities of states $\left(N_{c}, N_{\nu}\right)$ to vary with position. This allows heterostructure device simulation capabilities to be easily implemented [7], and hence permits the simulation of graded-base SiGe HBT's. The influence of Fermi-Dirac statistics has been accounted for through the Fermi correction factors $\left(\gamma_{n}, \gamma_{p}\right)$ [8], allowing a more physically correct simulation, especially at LNT. Advanced parameter models have been implemented in SCORPIO for accurately simulating the effects of carrier freezeout [9], Shockley-Read-Hall and Auger recombination [1], and apparent bandgap narrowing [10]. Doping and temperature dependent majority and minority carrier mobilities are calculated for both electrons and holes [11]. In addition, SCORPIO models the bandgap energy and the conduction and valence band densities of states as functions of germanium mole fraction [12], [13].

For numerical implementation, Eqs. (1)-(7) are discretized on a one-dimensional finite difference mesh. A one-dimensional device model is used instead of a more complex two- or three-dimensional model primarily because, for the bipolar transistor, a one-dimensional structure can successfully simulate much of the device behavior [14]. Additionally, the reduction in complexity of the simulator, and therefore the 
required execution time, aids in the use of the program as an interactive design tool. For increased numerical stability, the current density expressions, (4) and (5), are discretized using the Scharfetter-Gummel transformation [15]. Since the equations are nonlinear, an iterative solution technique such as Newton's method must be used.

\subsection{The Problem of Convergence at Low Temperature}

A difficulty associated with the classical Newton method is the tendency to overestimate the length of the actual correction step for a given iteration. This phenomenon is frequently termed overshoot, and may result in slow convergence (i.e., many iterations are needed to obtain a solution). This problem may be avoided by using a damping parameter to limit the size of each correction step, thus improving convergence [16]. SCORPIO updates this damping parameter after each iteration in an attempt to accelerate convergence.

For every bias condition, Newton's method requires an initial approximation to the solution. If this initial guess fails to sufficiently approximate the actual solution (i.e., if the guess does not lie in the solution's region of attraction) then Newton's method will fail to converge. By default, SCORPIO will first attempt to solve Poisson's equation, assuming zero current flow, to obtain the zero-bias solution. This solution is then passed on to the fully-coupled equation solver. The bias condition is incremented in small steps, using the solution for one bias point as the initial guess for the next bias point. SCORPIO assumes the solver has converged to a solution when the relative error of the Newton update vector becomes suitably small (usually less than $10^{-7}$ ). Convergence difficulty for a given simulation may sometimes be avoided by simply taking smaller bias steps.

Another reason that Newton's method may fail to converge is the loss of numerical precision that may occur due to subtracting nearly equal numbers using finite precision arithmetic. Double precision (8-byte) arithmetic does not provide sufficient accuracy to resolve the solution updates for a given iteration at LNT. The corrupted update vector that results may move the solution approximation for the next iteration outside of the actual solution's region of attraction, and Newton's method will fail to converge. The basic device equations contain both positive and negative exponential functions of temperature. For example, the intrinsic carrier concentration varies over about thirty orders of magnitude from $300 \mathrm{~K}$ to $77 \mathrm{~K}$. As a result, the system of equations being solved becomes more ill-conditioned, and the solution algorithm becomes increasingly sensitive to the effects of precision loss and other sources of numerical error. Thus, obtaining convergence becomes increasingly more difficult with decreasing temperature. To help alleviate this difficulty, critical calculations are performed with extended (16-byte) precision for temperatures below $150 \mathrm{~K}$. While the subroutines which use extended-precision arithmetic are slower than those that do not, the extra precision is necessary for converging to accurate low-temperature solutions [17]. At the present time, the lowest temperature for which SCORPIO's zero-bias Poisson solver can successfully converge for an advanced SiGe HBT profile is $20 \mathrm{~K}$.

For a 250 node mesh, a RT simulation with SCORPIO typically requires 5-10 Newton iterations per bias step, with up to $100 \mathrm{mV}$ bias steps possible for low injection. For high injection, $1 \mathrm{mV}$ bias steps may be required. The average execution time is approximately 0.3 seconds per bias step on a Sun SPARC-10 workstation. Convergence at LNT typically requires $10-20$ Newton iterations per bias step, with up to $50 \mathrm{mV}$ bias steps possible for low injection. For high injection, $1 \mathrm{mV}$ bias steps are usually required. The average execution time for LNT simulations is approximately 1.5 seconds per bias step.

\section{SIMULATION RESULTS}

To demonstrate SCORPIO's usefulness, the realistic doping profile shown in Figure 2 has been simulated at RT and LNT. Note that this profile has been taken from measured SIMS data of an actual state-of-the-art SiGe HBT [18]. This device has a measured current gain of 82 , a peak cut-off frequency of $59 \mathrm{GHz}$, and an unloaded ECL gate delay of $28 \mathrm{ps}$ at LNT. The arsenic concentration in the emitter has been assumed to be $15 \%$ electrically active, and the graded $\mathrm{SiGe}$ base has a peak germanium mole fraction of approximately $8 \%$. 


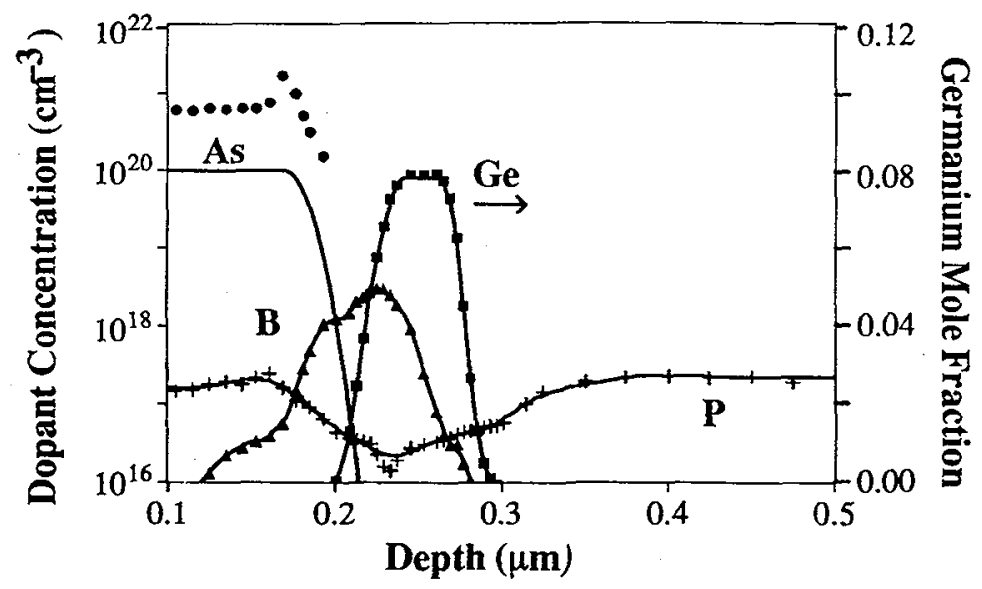

Figure 2. Doping profile used in simulator (solid line) compared to actual SIMS data (symbols).

To illustrate the effects of Ge on the device behavior, Figure 3 compares the simulated electrostatic potential in the base of this SiGe HBT to that of a silicon bipolar junction transistor (Si BJT) with identical doping. The smaller bandgap of the SiGe base enhances electron injection into the base. The bandgap grading produced by the germanium profile induces a drift field of about $20 \mathrm{kV} / \mathrm{cm}$ which enhances minority carrier transport through the base [19].

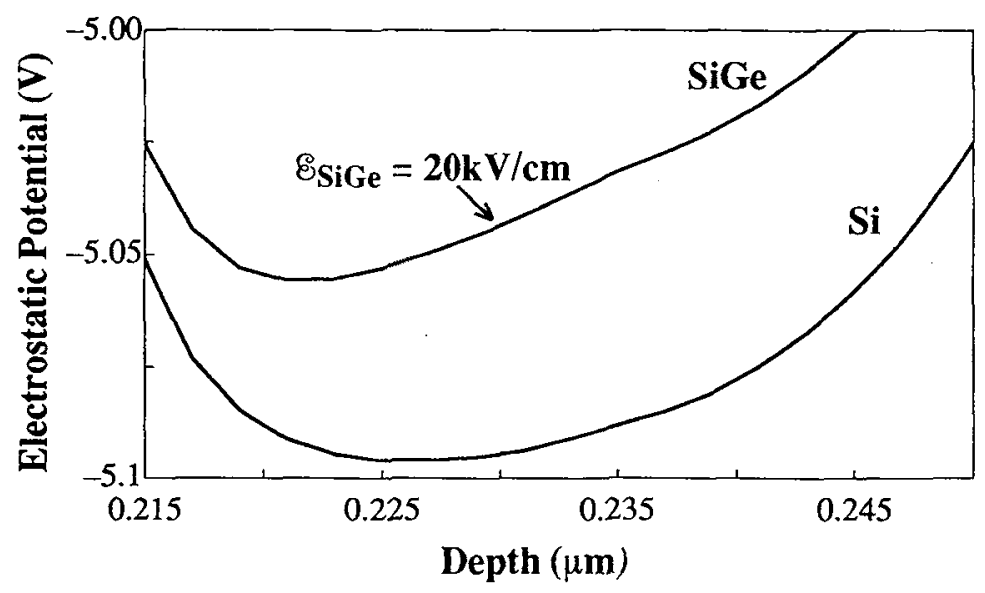

Figure 3. Simulated electrostatic potential in the base at $300 \mathrm{~K}$ for a SiGe HBT and a Si BJT.

As a first step towards model calibration, simulated values of the zero-bias pinched base sheet resistance $\left(R_{b i}\right)$ were compared to measured data. Reasonable agreement over temperature has been obtained, as shown in Figure $4 . R_{b i}$ increases with cooling due to carrier freezeout in the base. The effects of carrier freezeout can clearly be seen in Figure 5, which shows the simulated hole density in the base at $300 \mathrm{~K}$ and $77 \mathrm{~K}$. Figure 6 compares the simulated versus measured Gummel characteristics in the SiGe HBT at $310 \mathrm{~K}$ and $84 \mathrm{~K}$. The reasonable agreement obtained between the simulated and measured results indicates that the simulator is adequately modeling the dominant temperature dependencies in the device. Improved agreement with measured data is anticipated as further calibrations are made. 


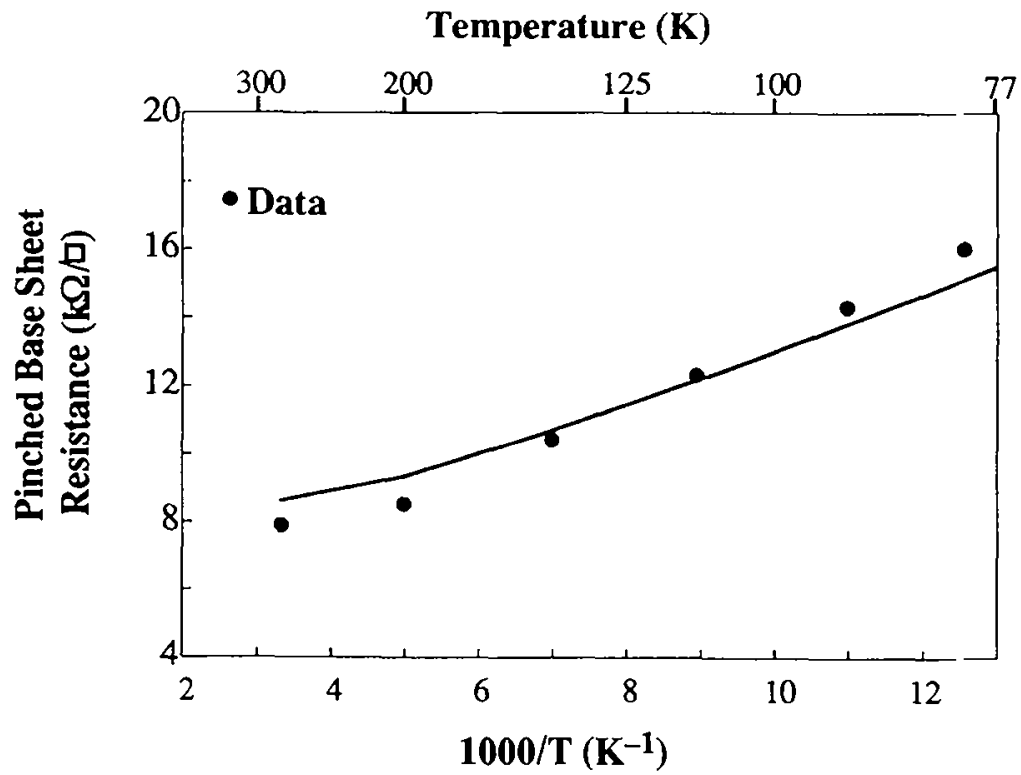

Figure 4. Simulated zero-bias pinched base sheet resistance vs. experimental data.

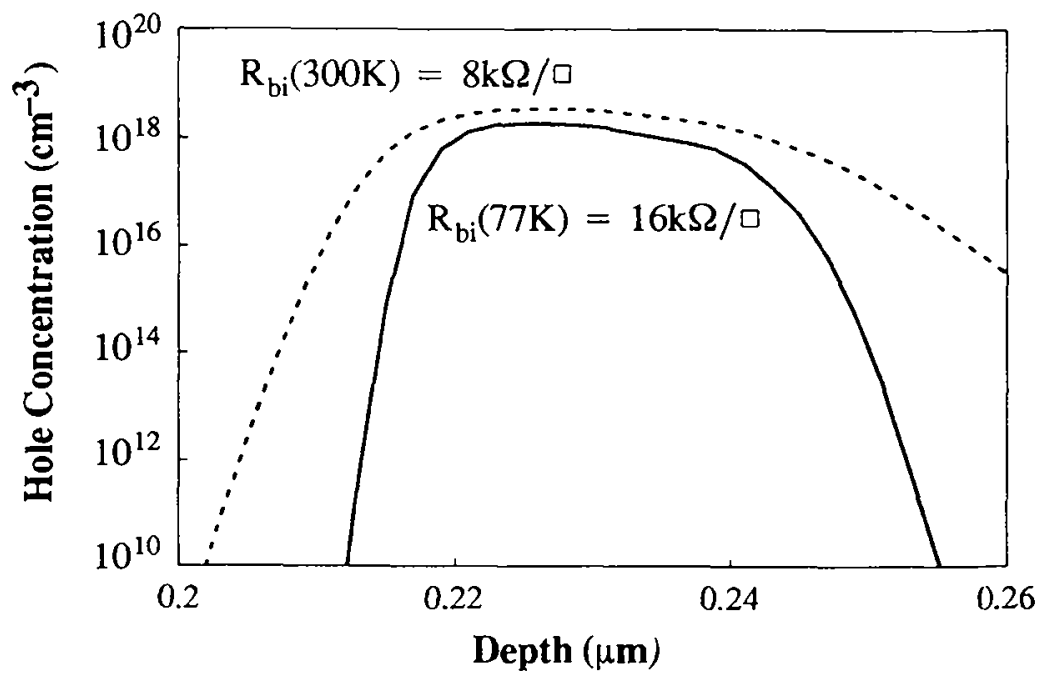

Figure 5. Simulated zero-bias hole concentration in the base at $300 \mathrm{~K}$ (dotted line) and $77 \mathrm{~K}$. 


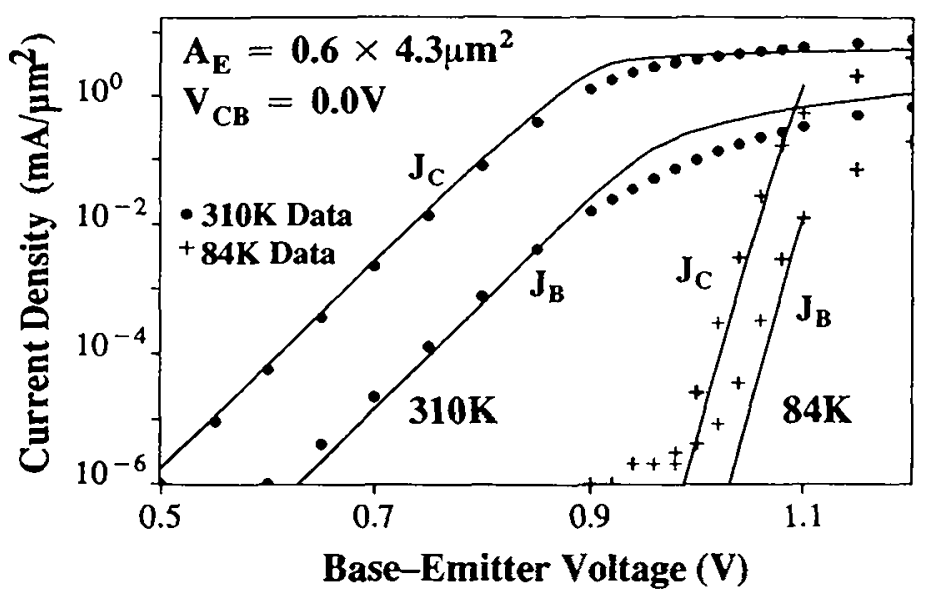

Figure 6. Simulated SiGe HBT $I-V$ characteristics vs. experimental data.

\section{SUMMARY}

SCORPIO, a simulation tool having robust convergence properties, has been developed for investigating $\mathrm{SiGe} \mathrm{HBT}$ behavior in the temperature range $77-400 \mathrm{~K}$. Strategic use of extended precision arithmetic and careful ordering of mathematical operations help provide rapid convergence at LNT with SCORPIO, even with advanced parameter models and realistic doping profiles. Comparisons of simulation results with experimental measurements are being used to ensure accurate model calibration. SCORPIO is intended to be a useful tool for device optimization and for investigating low-temperature device physics.

Acknowledgment - The authors would like to acknowledge E.F. Crabbé, J.H. Comfort, J.M.C. Stork, J.Y.-C. Sun, B.S. Meyerson, and the IBM Yorktown Silicon Facility for their contributions to this work. Portions of this work have been sponsored by the Alabama Microelectronics Science and Technology Center, Aubum University, AL, and by Analog Devices, Inc., Wilmington, MA.

\section{REFERENCES}

[1] Selberherr S., Analysis and Simulation of Semiconductor Devices (Springer Verlag Wien, NY, 1984).

[2] Chrzanowska-Jeske M. and Jaeger R.C., IEEE Tran. Elec. Dev. 36 (1989) 1475-1488.

[3] Stroustrup B., The C++ Programming Language (Addison-Wesley, MA, 1987).

[4] Markowich P.A. et al., Semiconductor Equations (Springer Verlag Wien, NY, 1990).

[5] Selberherr S., IEEE Tran. Elec. Dev. 36 (1989) 1464-1474.

[6] DeMari A., Solid-State Elec. 11 (1968) 33-58.

[7] Marshak A.H., Solid-State Elec. 31 (1988) 1551-1553.

[8] Lundstrom M.S. and Schueike R.J., IEEE Trans. Elec. Dev. ED-30 (1983) 1151-1159.

[9] Cole D.C. and Johnson J.B., Proc. of the IEEE Workshop on Low Temp. Semi. Elec. (1989) 73-77.

[10] Klaassen D.M.B., et al., Solid-State Elec. 35 (1992) 125-129.

[11] Klaassen D.M.B., Solid-State Elec. 35 (1992) 953-959 and 961-967.

[12] People R., IEEE J. Quantum Elec. 22 (1986) 1696-1710.

[13] Prinz E.J. et al., IEDM Tech. Digest (1989) 639-642.

[14] Kurata M., Numerical Analysis for Semiconductor Devices (Lexington Books, MA, 1982).

[15] Scharfetter D.L. and Gummel H.K., IEEE Trans. Elec. Dev. 16 (1969) 64-77.

[16] Bank R.E. and Rose D.J., Numerische Mathematik 37 (1981) 279-295.

[17] Johnson J.B. and Voldman S.H., COMPEL 10 (1991) 573-588.

[18] Cressler J.D. et al., IEEE Elec. Dev. Lett. 12 (1991) 166-168.

[19] Cressler J.D. et al., IEEE Tran. Elec. Dev. 40 (1993) 542-556. 